\author{
D. Krishna Kishor · Ranjan Ganguli · S. Gopalakrishnan
}

\title{
Uncertainty analysis of vibrational frequencies of an incompressible liquid in a rectangular tank with and without a baffle using polynomial chaos expansion
}

Received: 16 November 2010 / Published online: 1 April 2011

(C) Springer-Verlag 2011

\begin{abstract}
Polynomial chaos expansion (PCE) with Latin hypercube sampling (LHS) is employed for calculating the vibrational frequencies of an inviscid incompressible fluid partially filled in a rectangular tank with and without a baffle. Vibration frequencies of the coupled system are described through their projections on the PCE which uses orthogonal basis functions. PCE coefficients are evaluated using LHS. Convergence on the coefficient of variation is used to find the orthogonal polynomial basis function order which is employed in PCE. It is observed that the dispersion in the eigenvalues is more in the case of a rectangular tank with a baffle. The accuracy of the PCE method is verified with standard MCS results and is found to be more efficient.
\end{abstract}

\section{Introduction}

Sloshing phenomena are present in almost all moving vehicles that contain liquid storage tanks. In aircrafts, the fuel is stored in wings and horizontal tails which can create slosh motion during unsteady motions of the aircraft. The liquid fuel sloshing developed in these sections can couple with flexible wing vibration modes, and this can alter the flutter characteristics [1]. In addition, aircraft dynamic stability depends on the overall effect of the fuel sloshing in the wings [2]. A larger portion of the initial weight in launch vehicles will be in the form of liquid fuel. This liquid fuel during launch or lift-off creates large dynamic forces on the launch vehicle walls [3].

Liquid sloshing in partially filled containers is a complex phenomenon due to the random distribution of liquid masses. In the case of a completely filled tank, the liquid motion follows that of the container, and the liquid behaves like a solid body with a fixed center of mass. For containers of symmetric cross section, the lowest few frequencies corresponding to the first few antisymmetric mode shapes are of primary interest. The symmetric modes do not create any resultant lateral forces and moments [3]. In cylindrical containers during sloshing, the lower part of the liquid performs oscillations as though it was a rigid body and only the liquid in the vicinity of the free surface moves independently; as the frequency of oscillation of the free surface increases, the motion penetrates less deeply into the liquid. For liquid depth to diameter ratio, $h / d>1$, the liquid natural frequencies may be considered to be independent of the liquid depth [3-5].

Uncertainty is present in almost all systems in engineering and science. Sources of uncertainty in engineering are manufacturing imprecision, imperfect knowledge, variability associated with loading, material

D. K. Kishor · R. Ganguli $(\varangle) \cdot$ S. Gopalakrishnan

Department of Aerospace Engineering, Indian Institute of Science, Bangalore, India

E-mail: ganguli@aero.iisc.ernet.in

D. K. Kishor

E-mail: dkishor@aero.iisc.ernet.in

S. Gopalakrishnan

E-mail: krishnan@aero.iisc.ernet.in 
properties, geometric dimensions, and unsteadiness in nature. A significant change in the system response or performance can take place due to a small variation in environmental or design variables. Engineers can design systems that are more efficient, robust, and reliable by understanding the effect of uncertainty on the system performance. Catastrophic failures can result if uncertainty effects on the physical model are ignored [6-10]. An accurate representation of uncertainties for a given system is crucial because different uncertainty representations may yield different interpretations for the system considered.

Uncertainties can be classified into two categories: aleatory and epistemic [11-14]. Aleatory (Random) uncertainty is also called inherent or irreducible uncertainty. In most of the systems, these uncertainties are present due to random and unsteady loads, human factor, manufacturing imprecision, etc. Epistemic uncertainty is a reducible uncertainty that comes from lack of knowledge and experimental data. Epistemic uncertainty requires more attention and careful judgement, since it is viewed as reducible uncertainty as more information is gathered based on past experience. Commonly probability density function (PDF) and interval information (II) are used to represent aleatory and epistemic uncertainties. If the relative frequency of certain realizations in system performance for a given random variable is of interest, probability density function is best suited. On the other hand, interval information is used to represent upper and lower bounds of random variables when the information on the probability density function is not available [16].

Different probability and non-probability theories have been developed to handle aleatory and epistemic uncertainties effectively. Probabilistic theories include the stochastic finite element method (SFEM), First and Second-Order Reliability Method [15], and Monte Carlo (MC) method [16]. The SFEM is an extension of deterministic FEM for considering fluctuations of the structural properties and loads of the stochastic systems $[16,17]$. The most popular SFEMs are the perturbation technique developed by Kleiber and Hein [18] and the Karhunen-Loeve (KL) polynomial expansion scheme developed by Ghanem and Spanos [19-23]. For low probability of failures or for highly nonlinear responses, first and second-order reliability theories are not acceptable [16]. The Monte Carlo simulation (MCS) is known as a simple random sampling method or statistical trial method that make realizations based on randomly generated sampling sets for uncertain variables. In MC simulation, no restrictions on the random variable range are imposed and can have a large coefficient of variations. In [24], the authors have shown the usefulness of MCS in selecting the aircraft design parameters. Monte Carlo simulation is conducted to understand the behavior of the Ares I launch vehicle and to assist with its design [25]. Few other applications of MCS can be found in [26,27]. In [28], the authors discuss the capabilities of various probabilistic methods including the perturbation method, the polynomial chaos expansion, direct and advanced MCS, and the random matrix theory. It is mentioned in [28] that advanced MCS procedures prove to be the most versatile approach for the uncertainty analysis of large complex structures.

The non-probabilistic theories include interval analysis, Fuzzy theory, possibility theory, and evidence theory [29]. In [29], the authors have considered the modeling of uncertain structural systems using interval analysis. A fuzzy finite element approach can be used for imprecisely defined systems [30-33]. Since many engineering systems in practice are too complex to be defined in precise mathematical terms, they often contain information and features that are vague, imprecise, qualitative, or incomplete. The traditional deterministic and probabilistic techniques are not adequate for analyzing such systems. In [34-36], the authors have used possibility theory to assess design reliability with incomplete information. The possibility theory can be viewed as a variant of fuzzy set theory. Evidence theory [37-40] allows us to express partial beliefs when it is impossible or impractical to assess the complete probability distribution confidently.

As explained in the previous paragraphs, Ghanem and Spanos [19-23] introduced the spectral stochastic finite element method (SFEM). Since then, polynomial chaos expansion (PCE) has gained popularity as a technique for uncertainty quantification in a variety of applications due to its simplicity and easiness in implementation. This PCE is essentially a spectral expansion of the random variables using orthogonal basis functions [43]. In the PCE, orthogonal polynomials like Hermite, Legendre, and Laguerre polynomials are used as the basis functions. The PCE technique is well suited for the representation of Gaussian processes [44]. As mentioned in [41], probabilistic collocation method and MCS were used to calculate the coefficients of the PCE. Each of these methods has its own limitations in calculating the coefficients of the PCE. Wei et al. [42] constructed PCE with points of monomial cubature rules (MCR) and showed that PCE with MCR requires less sampling points, but the cubature formulae are precise for monomials up to a certain degree. Whereas, as shown in [41], the Latin hypercube sampling (LHS) method generates random sampling points that represent the total random space accurately and is computationally cheaper to generate. Here, the authors have used F statistics for the cut-off criteria, but it requires considerable experience about the cut-off rules. In the present paper, we follow a simple procedure to find the number of LHS points and the order of the orthogonal polynomials. Here, we use convergence of the mean and the coefficient of variation plots of the random system responses for the 
cut-off criteria. To the best knowledge of the authors, no probabilistic analysis has been carried out to study the effect of uncertainties on the acoustic fluid and fluid-structure vibration characteristics using polynomial chaos expansion using Hermite polynomials. This paper constitutes an exploratory work in this area.

In the present work, PCE is employed as a tool to study the variability/dispersion in the slosh and acoustic frequencies due to uncertainties in the material properties of the fluid and structure, and acceleration due to gravity. This study is important for the design of launch vehicle's (LV) control systems and elastic LV structures. From the practical applications perspective, liquid fuel amounts to a large portion of the initial weight in LVs, which creates substantial forces that are beyond capabilities of control systems to counteract. Since the LV is a complex dynamical system, the control system's natural frequencies, the elastic body frequencies, and the liquid fuel slosh frequencies must all be fairly widely separated to avoid coupling between various components [3]. Saturn-I launch vehicle's control frequency, fundamental slosh frequency, and fundamental bending frequencies are $0.4,0.6$, and $2 \mathrm{cps}$, respectively [3]. The liquid fuel may interact with both the control system dynamics and the elastic vehicle structural dynamics. Another practical application of the sloshing problem is in satellites carrying fuel onboard. The forces applied on the satellite which are needed for course corrections regularly will also induce sloshing motion. These sloshing motions affect the satellite motion as a whole due to their coupling with other control system parameters. An example of this problem can be found in [45], where NASA's NEAR (Near Earth Asteroid Rendezvous) satellite was delayed by 13 months due to sloshing of the liquid fuel onboard [46]. In such cases, the uncertainty studies of sloshing motion are important.

Two practical example problems that are common in aerospace, mechanical, and civil engineering fields are considered to study the effectiveness of the present method. In the first example problem, sloshing and acoustic frequencies in a rectangular tank partially filled with liquid are studied considering gravitational forces (g) $[47,48]$ and bulk modulus of the fluid $(\mathrm{K})[49]$ as random variables. In the second example problem, an interesting and complicated problem is considered. A rectangular tank partially filled with liquid having a baffle inside it is taken. The importance of each problem is discussed in the subsequent Sections along with results. The literature related to the numerical study of uncertainties in slosh and acoustic motions is very limited. Relatively little theoretical work relating the amplitude of the liquid motion to changing gravity has been documented. Traugott and Mehta [47] dealt in some generality with the important issue of wave phase with respect to the onset of the reduction in gravity. In [48], coupling of slosh dynamics with spacecraft attitude dynamics is investigated in response to the environmental disturbances (i.e., lateral impulse, gravity gradient, and g-jitter forces). The current work contributes to the knowledge base in this area.

The remainder of the paper is as follows. In Sect. 2, a deterministic Lagrangian finite element formulation used to model the linear acoustic fluids is discussed. In Sect. 3, a probabilistic model is presented using PCE with Latin hypercube samplings. Numerical examples and discussions are given in Sect. 4, followed by conclusions.

\section{Finite element formulation}

In this Section, we derive the finite element model for inviscid compressible fluid. An important behavior of the fluid system is its ability to displace without a change in volume. For bounded free surface fluid systems, the free surface of the fluid will move vertically by the so-called sloshing waves. These sloshing waves, in a steady-state condition, involve a harmonic interchange of the kinetic and potential energy of the fluid system. The low-frequency sloshing behavior of a fluid system involves incompressible modes of displacements which result in relatively large vertical surface displacements. The detailed finite element formulation of the present model is given in [50]. Here, we briefly give the FE formulation for the sake of completeness. The total potential energy of the fluid system $\left(\Pi_{P}\right)$ consists of the sum of the strain energy $\Pi_{E}$, due to compressibility and irrotationality, and the energy due to free surface oscillations of the fluid $\Pi_{S}$, which can be written as

$$
\Pi_{P}=\Pi_{E}+\Pi_{S} .
$$

Here

$$
\Pi_{E}=\frac{1}{2} \int_{\Omega}\{\varepsilon\}^{T}[\mathbf{C}]\{\varepsilon\} \mathrm{d} \Omega
$$

and

$$
\Pi_{S}=\frac{1}{2} \int_{S} \rho g\left\{u_{S}\right\}^{T}\left\{u_{s}\right\} \mathrm{d} S
$$


where $\{\varepsilon\}$ is the strain vector, and $\left\{u_{s}\right\}$ is the free surface displacement vector of the fluid on the surface $S, \rho$ is the mass density of the fluid, $g$ is the acceleration due to gravity, and $\Omega$ is the volume of the fluid domain. Matrix $[\mathbf{C}]$ is a diagonal matrix with bulk modulus $(\mathrm{K})$, rotational modulus $(\mathrm{R})$, and shear modulus $(\mathrm{G})$ as the diagonal terms. The kinetic energy $\left(\Pi_{T}\right)$ of the fluid is given by

$$
\Pi_{T}=\frac{1}{2} \int_{\Omega} \rho\{V\}^{T}\{V\} \mathrm{d} \Omega
$$

where $\{V\}$ is the velocity vector in Cartesian coordinates. The discrete form of the governing equations can be obtained by applying Hamilton's principle, which can be written as

$$
\delta \int_{t_{1}}^{t_{2}}\left(\Pi_{T}-\Pi_{P}+W_{n c}\right) \mathrm{d} t=0
$$

where $W_{n c}$ is the non-conservative energy due to applied forces and damping. We can express the displacements in $x, y$, and $z$ directions in terms of the nodal degrees of freedom using

$$
\left\{\begin{array}{c}
u \\
v \\
w
\end{array}\right\}=\left[\begin{array}{ccc}
{[N]^{T}} & {[0]} & {[0]} \\
{[0]} & {[N]^{T}} & {[0]} \\
{[0]} & {[0]} & {[N]^{T}}
\end{array}\right]\{d\}
$$

where $\{d\}^{T}$ is the nodal displacement vector. First, the energies are expressed in terms of the displacements by substituting the displacement fields given in Eq. (6) into Eqs. (2-4). These energies are in turn substituted into Eq. (5) and minimized to get

$$
[\mathbf{M}]\{\ddot{u}\}+[\mathbf{K}]\{u\}+\left[\mathbf{K}_{\mathrm{s}}\right]\left\{u_{s}\right\}=\{F\}
$$

where $\{\ddot{u}\}$ is the vector of nodal accelerations, $\left\{u_{s}\right\}$ is the displacement vector of free surface elements, and $\{F\}$ is the applied force vector. The mass matrix $[\mathbf{M}]$ and the stiffness matrix $[\mathbf{K}]$ are symmetric and banded, and they are given by

$$
[\mathbf{M}]=\int_{\Omega} \rho[\mathbf{N}]^{T}[\mathbf{N}] \mathrm{d} \Omega, \quad[\mathbf{K}]=\int_{\Omega}[\mathbf{B}]^{T}[\mathbf{C}][\mathbf{B}] \mathrm{d} \Omega .
$$

The sloshing stiffness matrix is given by

$$
\left[\mathbf{K}_{\mathrm{S}}\right]=\int_{S} \rho g\left[\mathbf{N}_{\mathrm{S}}\right]^{T}\left[\mathbf{N}_{\mathrm{S}}\right] \mathrm{d} S .
$$

The surface of the fluid is modeled as a two-dimensional plane element. The shape functions $\left[\mathbf{N}_{\mathrm{s}}\right]$ correspond to quadrilateral Lagrangian elements.

\section{Probabilistic model}

The current PCE approach that employs Latin hypercube samplings for obtaining the coefficients of PCE is discussed in the present Section. The hybrid procedure given in [41] is utilized here, and the modified steps are given as:

(1) Construct an approximate PCE using Hermite polynomials of order $n$.

(2) Select random variable design points using LHS.

(3) Calculate coefficients of PCE using design points selected through LHS.

(4) Conduct convergence test on the PCE constructed using coefficients calculated above.

(5) Conduct MCS analysis on the converged PCE. 
On the converged PCE, uncertainty analysis is carried out using MCS with random samples generated using any random number generator. Polynomial chaos expansion as discussed in [51] originates in Brownian motion, which represents the chaos present in the physical systems. Wiener [51] introduced a mathematical model of the Brownian motion using a multiple stochastic Lebesgue integral with homogeneous chaos. Since then, PCE has been used for the uncertainty analysis in several applications. The PCE, which employs orthogonal basis functions and is mean square convergent, is a good choice for estimating the response variability of uncertain systems. The general polynomial chaos expansion of a random process $w(\phi)$ can be represented as [19]

$$
\begin{aligned}
w(\phi)= & \left.c_{0} \Psi_{0}+\sum_{i_{1}=1}^{\infty} c_{i_{1}} \Psi_{1}\left[\xi_{i_{1}}(\phi)\right]+\sum_{i_{1}=1}^{\infty} \sum_{i_{2}=1}^{i_{1}} c_{i_{1} i_{2}} \Psi_{2}\left[\xi_{i_{1}}(\phi)\right) \xi_{i_{2}}(\phi)\right] \\
& \left.+\sum_{i_{1}=1}^{\infty} \sum_{i_{2}=1}^{i_{1}} \sum_{i_{3}=1}^{i_{2}} c_{i_{1} i_{2} i_{3}} \Psi_{3}\left[\xi_{i_{1}}(\phi)\right) \xi_{i_{2}}(\phi) \xi_{i_{3}}(\phi)\right]+\cdots+\cdots
\end{aligned}
$$

where $\Psi_{p}\left[\xi_{i_{1}}, \xi_{i_{2}}, \ldots, \xi_{i_{p}}\right]$ denotes the Hermite polynomial of order $p$, a $p$ dimensional polynomial of $\xi_{i}$, where $\xi_{i}$ are standard random variables, $\phi$ is the realization of these variables, and $c_{i_{p}}$ are the deterministic coefficients. Equation (10) can be written compactly for notational convenience as

$$
w(\phi)=\sum_{i=0}^{\infty} a_{i} \Phi_{i}[\xi(\phi)]
$$

where the coefficients and basis functions in Eqs. (10) and (11) are similar.

The orthogonality property of the Hermite polynomials used in the polynomial chaos expansion in Eq. (10) can be written as

$$
\left\langle\Phi_{i} \Phi_{j}\right\rangle=\delta_{i j}\left\langle\Phi_{i}^{2}\right\rangle
$$

where $\delta_{i j}$ is the Kronecker delta and $\langle\cdot\rangle$ is the expectation of the weighted inner product of these polynomials in variable $\xi$. The general expression of the Hermite polynomials can be written as

$$
\Phi_{p}\left[\xi_{i_{1}}, \xi_{i_{2}}, \ldots \xi_{i_{p}}\right]=(-1)^{p} e^{\left(\frac{1}{2} \xi^{T} \xi\right)} \frac{\partial^{p} e^{\left(-\frac{1}{2} \xi^{T} \xi\right)}}{\partial \xi_{i_{1}} \ldots \partial \xi_{i_{p}}}
$$

where the vector $\xi$ consists of $n$ standard random variables.

In the uncertainty analysis, few input variables are considered as random variables. Hence, the output $y$ will also be random corresponding to the assumed input random variable and its probability density function. The random output $y$ can be written as a convergent PCE with orthogonal polynomials as

$$
y(\xi)=\sum_{i} \beta_{i} \Phi_{i}(\xi)
$$

The coefficients $\beta_{i}$ in the above equation represent the complete description of the system response $y$ due to random variables $\xi$ present in the model. For obtaining complete information of the system random response $(y)$, the coefficients of the PC expansion have to be calculated efficiently. There exist two classical methods for this purpose: the intrusive and non-intrusive method, respectively. The intrusive method requires modifications of the numerical code and is sometimes in few problems difficult to implement. Whereas, the non-intrusive method is easy to implement, which requires only output ( $y$ ) of the numerical code (like FEM) for each input random variable $(\xi)$. Various non-intrusive methods are discussed in [52]. As mentioned in [41], MCS can be used for calculating the PCE coefficients in the non-intrusive method but requires a large number of realizations for accurate results. Latin hypercube sampling (LHS) that showed promising results [41] for this kind of analysis is employed in the present analysis. Once the input random samplings are generated using LHS for given random variables, the PCE coefficients can be calculated using the least squares method. Here, for each input random sample, the output $(y)$ from the numerical code can be obtained which will be used subsequently 
in the least squares method. The coefficients of the Eq. (14) $\beta=\left(\beta_{0}, \beta_{1}, \ldots \beta_{p}\right)^{T}$ will be an approximation $\hat{\beta}$ due to truncation of the expansion. It can be written as

$$
\hat{y}(\xi)=\sum_{i}^{p} \hat{\beta}_{i} \Phi_{i}(\xi)
$$

where $p$ is the order of the truncated Hermite orthogonal polynomials. Now, taking least squares of the truncated series, we get

$$
\varepsilon=\sum_{j=1}^{m}\left(y^{(j)}-\hat{y}\right)^{2}
$$

where $\varepsilon$ represents the error and $m$ represents the number of sampling points generated using LHS for random variables. Minimizing Eq. (16) with respect to $y$ and $\hat{\beta}$, the PCE coefficients can be obtained as

$$
\hat{\beta}=\left(\Gamma^{T} \Gamma\right)^{-1} \Gamma^{T} Y
$$

where matrices $Y, \Gamma$, and $\hat{\beta}$ can be written as

$$
\{Y\}=\left[\begin{array}{l}
y_{1} \\
y_{2} \\
\cdot \\
\cdot \\
\cdot \\
y_{k}
\end{array}\right],\{\Gamma\}=\left[\begin{array}{ccccc}
1 & \Phi_{1}\left(\xi_{1}\right) & \Phi_{2}\left(\xi_{1}\right) & \ldots & \Phi_{k}\left(\xi_{1}\right) \\
1 & \Phi_{1}\left(\xi_{2}\right) & \Phi_{2}\left(\xi_{2}\right) & \ldots & \Phi_{k}\left(\xi_{2}\right) \\
\cdot & \cdot & \cdot & . & \cdot \\
\cdot & \cdot & \cdot & \cdot & \cdot \\
\cdot & \cdot & \cdot & \cdot & \cdot \\
1 & \Phi_{1}\left(\xi_{n}\right) & \Phi_{2}\left(\xi_{n}\right) & \ldots & \Phi_{k}\left(\xi_{n}\right)
\end{array}\right],\{\hat{\beta}\}=\left[\begin{array}{l}
\beta_{0} \\
\beta_{1} \\
\cdot \\
\cdot \\
\cdot \\
\beta_{k}
\end{array}\right]
$$

After an approximate model for the system response is constructed as given in Eq. (15), the convergence tests on the system response mean and coefficient of variation are performed to check whether a sufficient fit has been achieved.

\section{Numerical results}

In this Section, we use the PCE formulation developed in the previous Section to study the variability in vibrations of the acoustic fluids due to random input variables considered. Two practical problems that are commonly experienced in many aerospace, mechanical, and civil engineering fields are considered. The first problem involves fluid sloshing and acoustic motions in a rectangular tank without a baffle. The second problem addresses the same fluid motion in a rectangular tank with a baffle inside it.

\subsection{Uncertainties in slosh and acoustic frequencies in a rectangular tank}

In this example problem, we consider a rectangular tank partially filled with liquid as shown in Fig. 1. The liquid can exhibit low-frequency slosh modes and high-frequency acoustic modes. As explained in the introduction, antisymmetric slosh modes are important since these modes create lateral forces on the tank walls. The acoustic modes are volume change modes and create forces on the bulk of the tank. In the present case, we have to consider the interaction between the liquid and structure motions. The finite element formulation developed in [50] works well for these kinds of problems. We make use of this fluid finite element code in the present uncertainty analysis.

The dimensions of the tank, fluid properties and finite element discretization of the whole system are given in Table 1 and in Fig. 1, respectively. The fluid domain is modeled with 125 consistent 8-noded hexahedral elements and the rectangular tank with 166 brick 8-noded elements, and each node has three degrees of freedom (DOF). It has been proved in [50] that the present FE model for the liquid can predict accurate results. In [50], the liquid in a rigid rectangular tank having the dimensions considered in the present paper is discretized with 512 consistent eight-noded elements. This particular problem has an exact solution for the vibrational frequencies. The error in the first slosh frequency obtained from FE solution with 512 elements is $1.1704 \%$. In the present paper, 125 consistent eight-noded elements are considered for the liquid discretization, and the 
Table 1 Fluid properties and rectangular tank dimensions

\begin{tabular}{lc}
\hline Fluid dimensions and properties & \\
\hline Length (l1) & $5.08 \mathrm{~m}$ \\
Width (b1) & $4.08 \mathrm{~m}$ \\
Height (h1) & $1.905 \mathrm{~m}$ \\
Bulk modulus (K) & $2.23 e 09 \mathrm{~N} / \mathrm{m}^{2}$ \\
Rotational modulus (R) & $2.23 e 12 \mathrm{~N} / \mathrm{m}^{2}$ \\
Fluid density ( $\rho$ ) & $1000 \mathrm{~kg} / \mathrm{m}^{3}$ \\
Acceleration due to gravity (g) & $9.8 \mathrm{~m} / \mathrm{s}^{2}$ \\
\hline Tank dimensions and properties & $5.3848 \mathrm{~m}$ \\
\hline Length (l) & $4.3848 \mathrm{~m}$ \\
Width (b) & $2.01 \mathrm{~m}$ \\
Height $(\mathrm{h})$ & $285 \mathrm{e} 08 \mathrm{~N} / \mathrm{m}^{2}$ \\
Young's modulus (E) & 0.2 \\
Poisson's ratio $(v)$ & $2,500 \mathrm{~kg} / \mathrm{m}^{3}$ \\
Mass density $(\rho)$ &
\end{tabular}

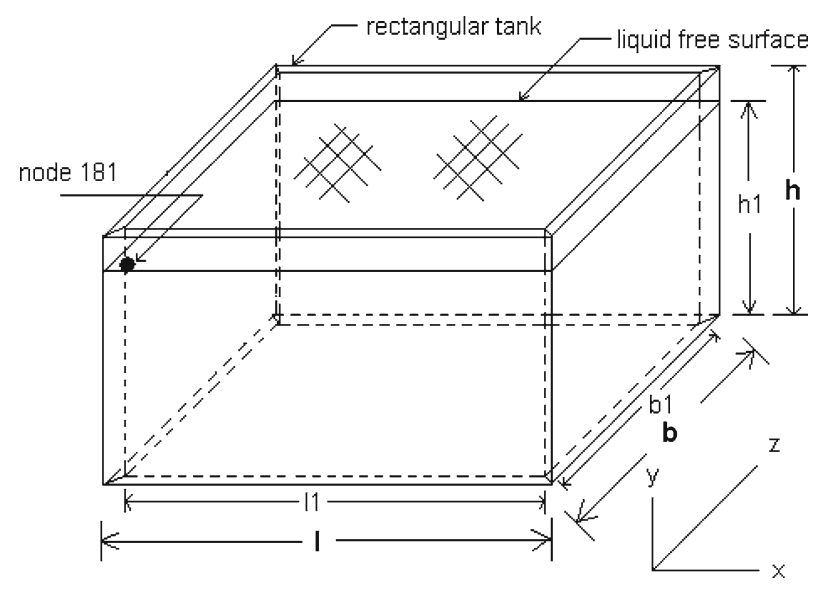

Fig. 1 3-D rectangular tank without a baffle

numerical error is $2.3140 \%$, and we believe that this accuracy is sufficient for the engineering practice. The FE model with 512 eight-noded elements requires enormous computational time for doing MCS, which is considered here as a benchmark validation case for the present PCE technique. The flexible tank is discretized with 166 eight-noded consistent brick elements, which were developed by Satish and Prathap ([56]). These consistent eight-noded brick elements are free of locking due to shear and Poisson's ratio; moreover, this element is also frame invariant. This number is obtained from the FE discretization of the liquid (125 elements) by maintaining the compatibility of displacements at the fluid-solid interfaces on all four sides. The error in the acoustic frequency obtained from an FE solution with 125 elements is $0.421 \%$. In addition, in [50], it has been shown that the liquid displacements at the free surface in rigid and flexible tanks do not differ much. Hence, the FE discretizations considered here are sufficient for accurate numerical solutions. The consistent fluid finite element developed in [50] calculates slosh and acoustic frequencies without generating any zero energy modes. This reduces the computational cost of the large systems. The coupled system has a total of 1488 degrees of freedom (DOF) in the finite element model. The coupling between the structure and the fluid motions is performed by writing the constraint equations between structural and fluid normal displacements as

$$
u_{f_{i}}-u_{s_{i}}=0, \quad i=1,2,3, \ldots . n
$$

where $u_{f}$ and $u_{s}$ are the normal displacements of the fluid and solid domains at the interface, respectively, and $n$ is the number of nodes on the interface. The first slosh mode shape from the coupled FE model is shown in Fig. 2. It is clearly seen from Fig. 2 that the first slosh mode is an antisymmetric mode in a rectangular tank without a baffle, which causes lateral forces on the tank. The significance of this antisymmetric slosh mode shape profile will be understood when we consider a baffle inside it as given in Sect. 4.2. The eigenvalues of this coupled system depend on the geometry of the tank, density and bulk modulus of the fluid, and the 


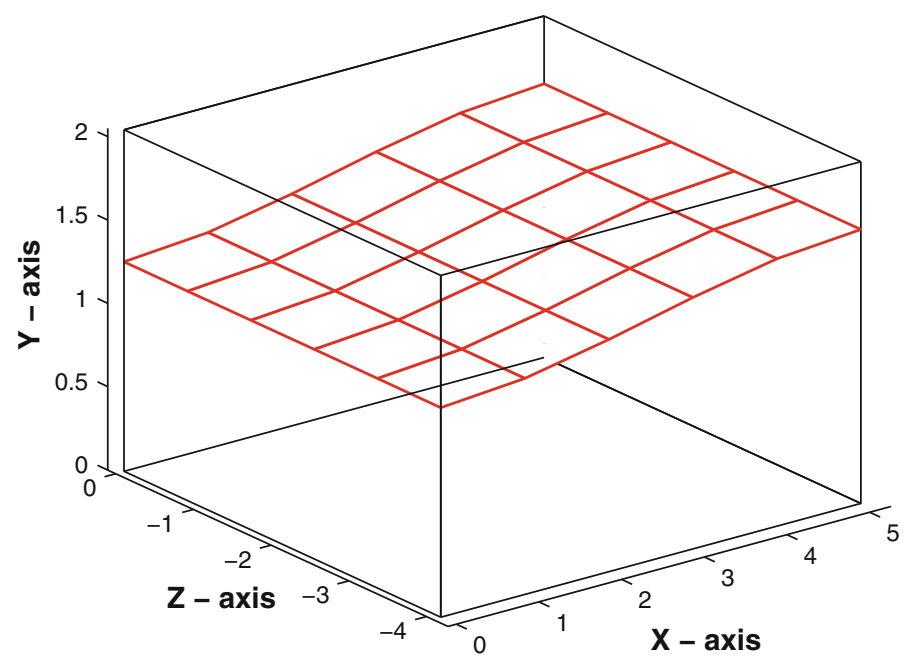

Fig. 2 First slosh mode shape in a rectangular tank without a baffle

acceleration due to gravity. In a space vehicle, the fuel storage tank will have a fixed geometry and the fuel height in the tank will reduce as time progresses, and it also experiences lateral impulses, gravity gradient forces, and g-jitter forces [48]. In a typical aircraft flight envelope, the slosh frequencies undergo variations corresponding to the variations in the gravitational forces and in the height of the fuel column.

In the present study, we consider the gravitational forces and bulk modulus of the fluid as the two independent random variables. For a typical transport aircraft, "g" varies from $-1.0 \mathrm{~g}$ to $2.5 \mathrm{~g}$ in the flight envelope. To get the actual variation in "g" and its distribution for an aircraft, one has to depend on the flight measurement data. Getting such data is not straight forward, so, we consider a Gaussian distribution with mean " $1 \mathrm{~g}$ " and coefficient of variation 0.1 . We believe that this approximation fairly accounts for all the uncertainties related to gravitational forces present in the actual flight envelope. For the case of bulk modulus of the fluid, a value of 0.05 is taken for the coefficient of variation.

While fitting the regression model for this problem, LHS is employed to calculate the coefficients of PCE. Generally, a lower-order model is preferred to a higher-order model in regression analysis because an arbitrary fit of higher-order polynomials may create serious errors [41]. Therefore, we present a simple convergence criterion to check the order of the polynomials by calculating the mean and coefficient of variation of the random system response. The details are given along with results in the subsequent Sections. We can initially set the approximate model of $Y$ with one random input variable $(\xi)$ by introducing the second-order Hermite polynomials as

$$
\hat{Y}=\beta_{0} \Psi_{0}(\xi)+\beta_{1} \Psi_{1}(\xi)+\beta_{2} \Psi_{2}(\xi)
$$

where $\xi$ has a standard normal distribution with mean 0 and standard deviation 1 . Here, $\Psi_{i}$ 's are the polynomial basis functions, which are orthogonal Hermite polynomials as given in Sect. 2. In the present regression model, $\hat{Y}$ represents the slosh and acoustic frequencies, respectively, corresponding to the random variables considered: gravitational forces and bulk modulus of the fluid. Here, while considering the gravitational forces as the random input variables, the bulk modulus of the fluid is taken as the fixed quantity, and similarly when the bulk modulus of the fluid is taken as the random input variable, the gravitational force is considered as a fixed quantity. The number of coefficients ( $\beta_{0}, \beta_{1}, \beta_{2}$, etc.) depends on the number of random input variables and the order of the Hermite polynomials. To find the unknown coefficients $\beta_{0}, \beta_{1}$, and $\beta_{2}$ of the approximate model $\hat{Y}$, LHS is used to identify the input random design points(acceleration due to gravity $\mathrm{g}$ and bulk modulus $\mathrm{K})$. The number of design points must be higher than the number of unknown coefficients [41].

A total of 5, 10, and 20 LHS samples are considered, and the regression models $(\hat{Y})$ are constructed corresponding to the Hermite polynomial order $(p)$. Once the regression models $(\hat{Y})$ are constructed, convergence tests on the mean and the coefficient of variation of the first slosh and acoustic frequencies are carried out to check the accuracy of the fitted regression model. While calculating the system responses (eigenvalues), a total of 5500 normal random sampling points is considered. The results of the convergence tests are given in Tables 2 and 3 and in Fig. 3. From these two Tables, we can conclude that the variation in the mean of the 
Table 2 Convergence of the first slosh frequency with Hermite polynomial orders

\begin{tabular}{lllll}
\hline LHS samples & $p=1$ & $p=2$ & $p=3$ & $p=4$ \\
\hline 5 & 2.28872 & 2.2879 & 2.2880 & 2.2880 \\
10 & 2.28853 & 2.2880 & 2.2880 & 2.2880 \\
20 & 2.28775 & 2.2879 & 2.2880 & 2.2880 \\
\hline
\end{tabular}

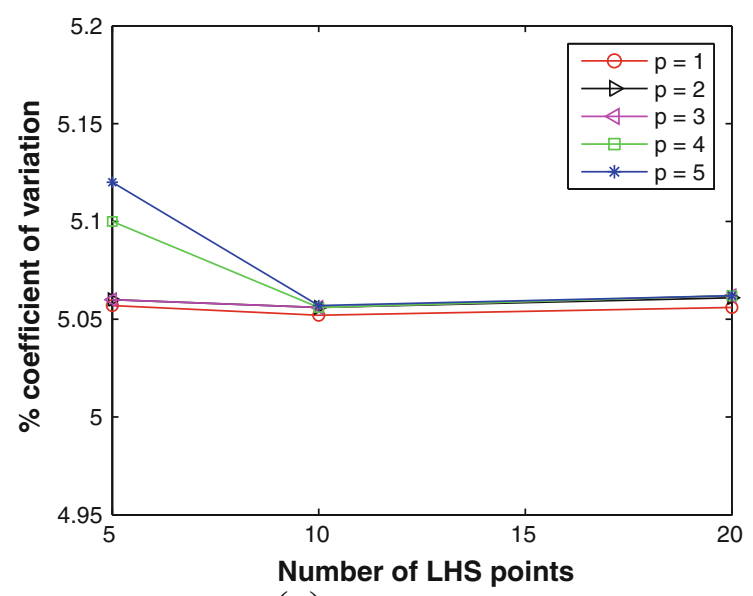

(a) First slosh frequency

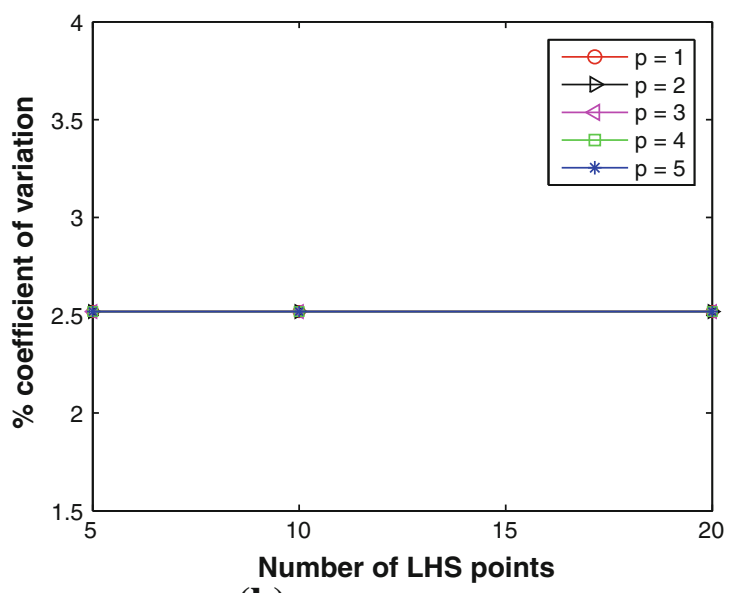

(b) First acoustic frequency

Fig. 3 Convergence of coefficient of variation with number of LHS points for various polynomial orders

Table 3 Convergence of the first acoustic frequency with Hermite polynomial orders

\begin{tabular}{lllll}
\hline LHS samples & $p=1$ & $p=2$ & $p=3$ & $p=4$ \\
\hline 5 & 1236.3 & 1236.2 & 1236.1 & 1236.1 \\
10 & 1236.1 & 1236.1 & 1236.1 & 1236.1 \\
20 & 1236.1 & 1236.1 & 1236.1 & 1236.1 \\
\hline
\end{tabular}

random system responses does not change significantly from the Hermite polynomial order $p=3$ to $p=4$ with the number of LHS points larger than 10 as given Table 2 and larger than 5 in Table 3 . Generally, the mean of any random system response will converge quickly. In order to find the correct PCE polynomial order and the number of LHS points, we have plotted in Fig. 3a the coefficient of variation of the first slosh frequency with the number of LHS points for various Hermite polynomial orders $(p)$. From Table 2 and Fig. 3a, it can be concluded that the regression model constructed with polynomial order $p=4$ and 20 LHS points is sufficient. Once the best fit for the regression model is obtained, the accuracy of the PCE is verified by comparing the probability of occurrence with the standard MCS results. Typically, MCS results are taken as the standard benchmark validation results. While constructing the MCS results, 5,500 random samples with mean and coefficient of variation considered previously are taken. In the case of PCE results, 5,500 and 10,000 random samples are considered. Here, we have shown the PCE results obtained from 10,000 samples only, since the differences between these two sampling sets are negligible. As shown in Fig. 4, probabilities of occurrence distributions from PCE and MCS are in good agreement.

In the case of acoustic frequencies, as seen from the Table 3 and Fig. 3b, mean and coefficient of variation values converge quickly at low polynomial orders and for a smaller number of LHS sampling points. The best regression model is constructed again with $p=4$ and 20 LHS points. After constructing the regression model, the probability of occurrence of the acoustic frequency is compared with MCS results in Fig. 5. Here again, while constructing the PCE and MCS solutions, the same number of samples as taken in the previous case is considered. Figures 4 and 5 show the accuracy and efficiency of the PCE approach for the probabilistic analysis of slosh and acoustic frequencies, respectively. From the above analysis, the first slosh and acoustic frequencies exhibited 5.08 and $2.5 \%$ of the coefficient of variations, respectively, for 10 and $5 \%$ coefficient of variations taken in the acceleration due to gravity and bulk modulus of the fluid. The dispersion in the system eigenvalues is considerably small corresponding to the uncertainties considered. Moreover, the probabilities of occurrence distributions are symmetric about the mean values. 


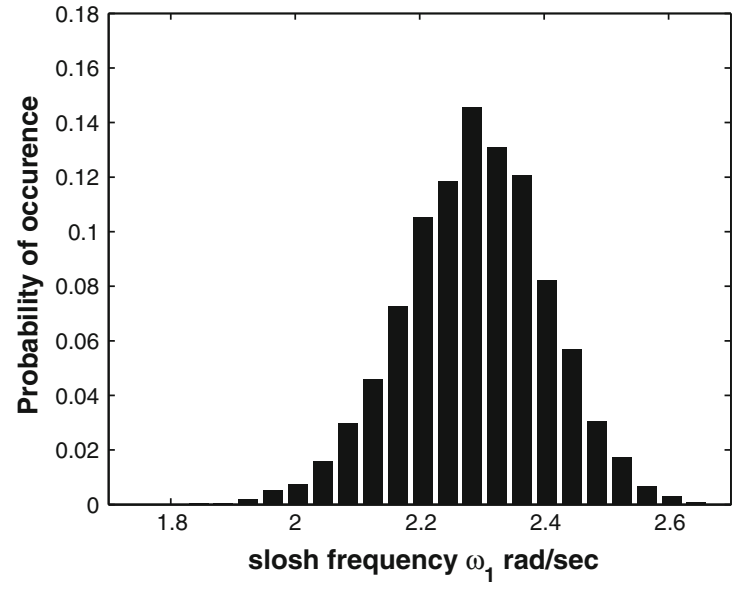

(a) PCE results

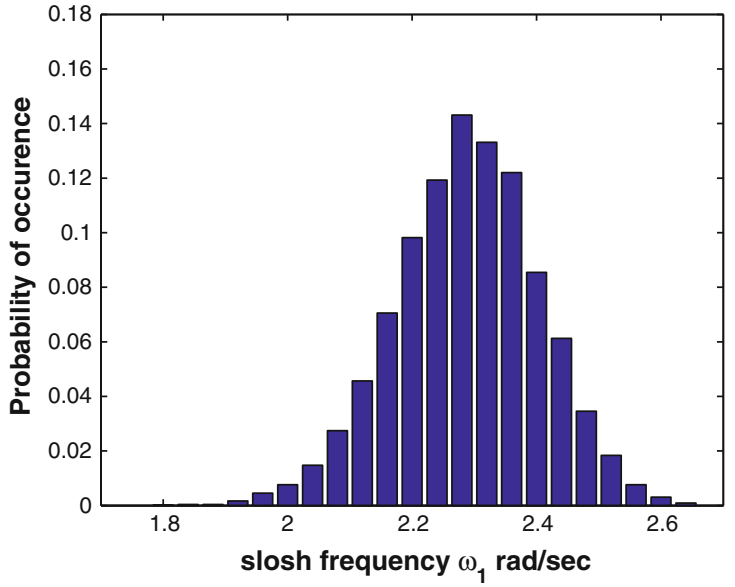

(b) MCS results

Fig. 4 Probability of occurrence distributions of the first slosh frequency $\omega_{1}$

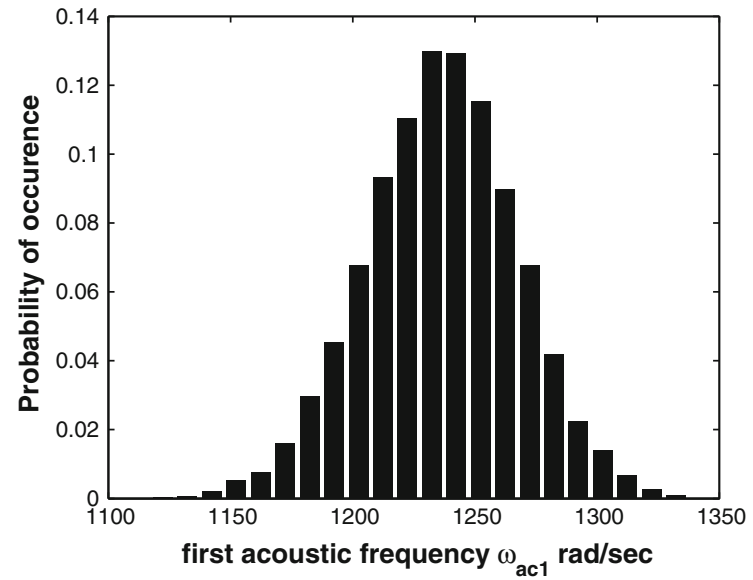

(a) PCE results

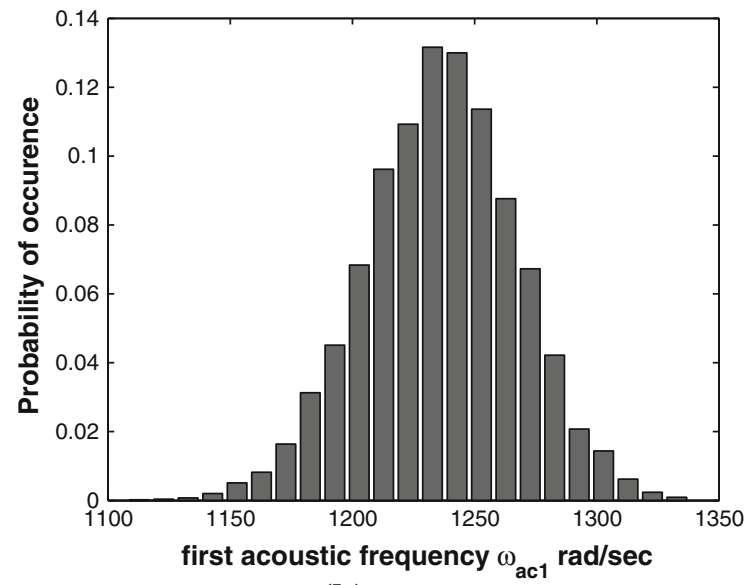

(b) MCS results

Fig. 5 Probability of occurrence distributions of the first acoustic frequency $\omega_{1}$

\subsection{Uncertainties in slosh and acoustic frequencies in a rectangular tank with a baffle}

In the second example problem, we consider a rectangular tank partially filled with liquid, which also contains a baffle inside the tank as shown in Fig. 6. The dimensions of the tank are given in Table 1, and the baffle dimensions are length $l 2=3.048 \mathrm{~m}$, the width of the plate is $0.816 \mathrm{~m}$, and the thickness is $0.1 \mathrm{~m}$. The distance between the baffle and bottom (h2) is $0.762 \mathrm{~m}$, and the side (b2) as shown in Fig. 6 is $1.632 \mathrm{~m}$. The flexible baffle is considered to be made of duraluminium with $E=62.43 e 9 P a$ and $v=0.35$ [55].

In the FE discretization of the model shown in Fig. 6, the same number of brick elements used in Sect. 4.1 is used for the rectangular tank. While modeling the liquid and the baffle, 119 consistent 8-noded fluid elements and six consistent brick elements [56] are taken, respectively. The FE model has a total of 1448 DOFs. The accuracy of the present FE model for this fluid-structure configuration is verified through a convergent test on the vibrational frequencies. Convergence test results of the first slosh frequency are shown in Fig. 7. From Fig. 7, the first slosh frequency with 119 and 169 consistent fluid elements is $5.0507 \mathrm{rad} / \mathrm{sec}$ and 5.0798 $\mathrm{rad} / \mathrm{sec}$, respectively. The difference in the slosh frequencies between these two FE discretizations is only $0.576 \%$. This shows that the considered FE discretization (119 elements) is a converged one for vibration analysis. Therefore, we have considered 119 FEs, since the computational cost is much less compared to 169 FEs. This FE model is employed in the subsequent analysis. The importance of this configuration is found in [3] and discussed here briefly. 


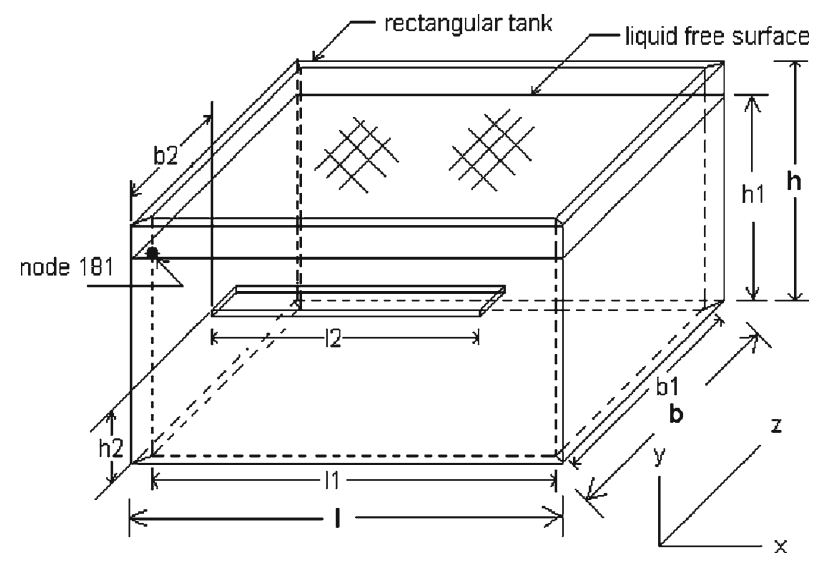

Fig. 6 3-D rectangular tank with a baffle

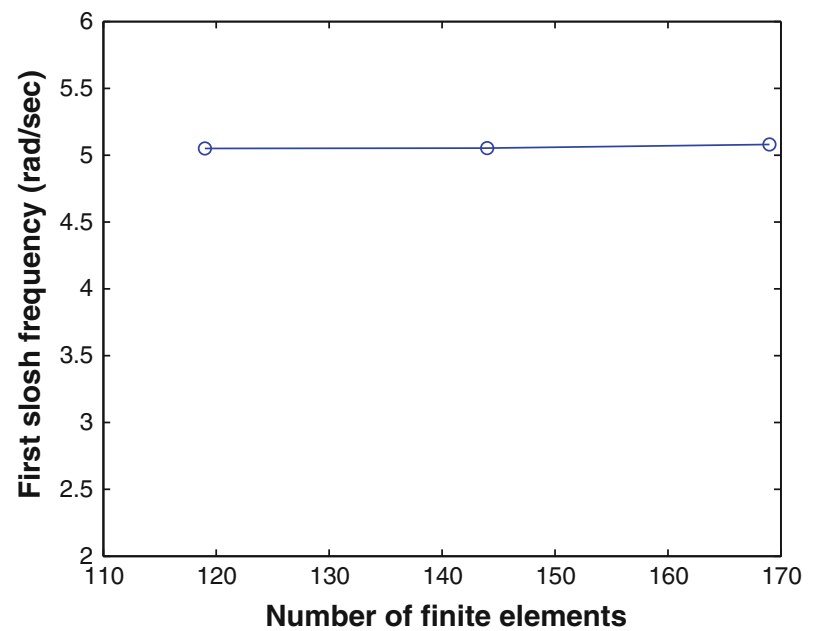

Fig. 7 Convergence test on the first slosh frequency in a rectangular tank with a baffle

The liquid, oscillating in its fundamental mode, produces a wave having its maximum amplitude at the wall. The direction of the flow in the vicinity of the wall is essentially vertical and thus normal to the baffle located at a distance below the free surface. Baffles that have a tendency to transfer the first-mode sloshing into a rotary motion have been briefly investigated in [53]. A number of studies have suggested that flexible baffles may offer substantial advantages in terms of both increased damping effectiveness and reduced baffle weight [3]. Of various baffle configurations examined by Silveira et al. [54], the elastic cantilever configuration was found to be superior due to its high damping and very low weight characteristics. The damping on the liquid sloshing motion depends on various parameters, such as gravitational acceleration, liquid kinematic viscosity, liquid depth, baffle size, and distance of the baffle from the free surface [3].

In the present paper, attention is focused on the dispersion in the first few slosh frequencies due to considered random processes. Since baffles are commonly employed in launch vehicles to dampen the liquid sloshing motion and shift the fundamental slosh frequencies [53,54], they require careful analysis. As discussed in the introduction, liquid sloshing frequencies, control system natural frequencies, and the elastic body frequencies must be fairly widely separated in launch vehicles to avoid the coupling between various components. This is not always straight forward, and introducing baffles in the liquid propellant tank complicates the system eigenvalue behavior. In such cases, the dispersion study in the fundamental slosh frequency is very important from the design point of view in avoiding coupling. In the present example problem, consistent with the earlier analysis, acceleration due to gravity, bulk modulus of the fluid, and Young's modulus of the flexible baffle are considered as independent random processes. The authors believe that the present example problem will definitely throw some light on this interesting area from the uncertainty point of view. Here, four cases are considered to isolate the uncertainty effects in the slosh and acoustic frequencies. The first case considers 


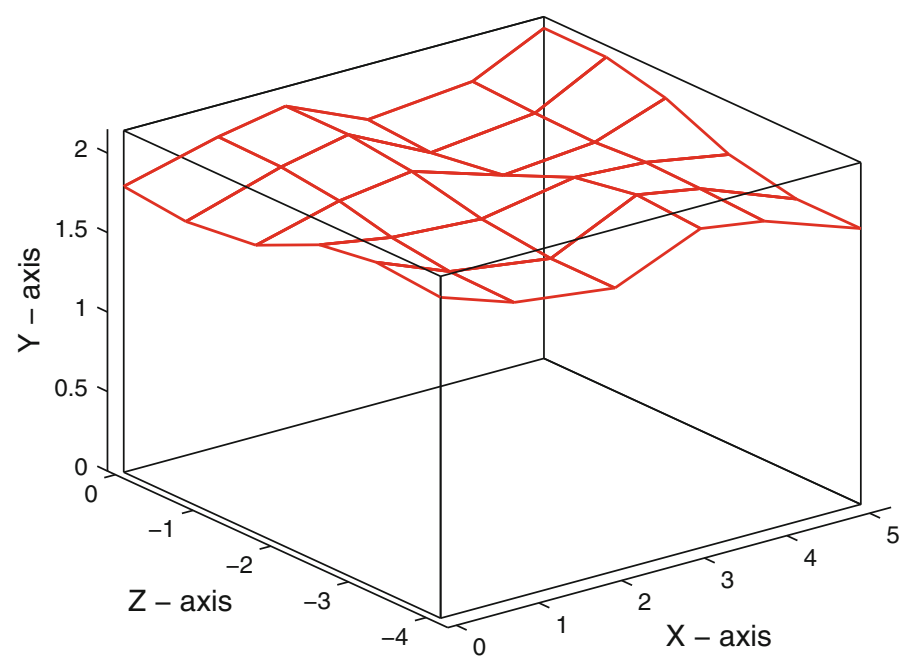

Fig. 8 First slosh mode shape in a rectangular tank with a baffle

Table 4 Convergence of the first slosh frequency with Hermite polynomial orders

\begin{tabular}{rllll}
\hline LHS samples & $n=1$ & $n=2$ & $n=3$ & $n=4$ \\
\hline 20 & 5.0055 & 5.0049 & 5.0018 & 5.0020 \\
50 & 5.0054 & 5.0053 & 5.0067 & 5.0107 \\
100 & 4.9866 & 4.9865 & 4.9873 & 4.9870 \\
200 & 4.9093 & 4.9019 & 4.9011 & 4.9011 \\
\hline
\end{tabular}

acceleration due to gravity $(\mathrm{g})$ as a random variable. The second case considers the bulk modulus of the fluid as random variable. Third case considers Young's modulus of the baffle as random variables, and in the fourth case, acceleration due to gravity, bulk modulus of the fluid, and Young's modulus of the baffle are considered as uncorrelated random variables.

\subsubsection{Case 1:}

In the first case, acceleration due to gravity ( $\mathrm{g}$ ) is considered as a random variable with mean " $1 \mathrm{~g}$ " and coefficient of variation (c.o.v.) 0.1. The first slosh vibration frequency mode in the rectangular tank with a baffle is given in Fig. 8. It is clearly seen that the antisymmetric nature of the slosh mode as seen in Fig. 2 is lost in Fig. 8. This behavior could be because of the baffle, which has a tendency to transfer the slosh mode into a rotational mode. This vibration mode shape is given here to better understand the significance of the baffle and its influence on the slosh mode shapes. This first slosh vibration mode is studied in the present Section and later, while dealing with uncertainties.

A total of 20, 50, 100, and 200 LHS samples are considered, and the regression models $(\hat{Y})$ are constructed corresponding to the Hermite polynomial orders $(p)$. Once the regression models $(\hat{Y})$ are constructed, convergence tests on the mean and coefficient of variation of the first slosh frequency are carried out to check the accuracy of the regression model. Convergence of mean and coefficient of variation of the first significant slosh frequency is given in Table 4 and in Fig. 9. Based on the results in Table 4 and Fig. 9, a regression model is constructed using polynomial order $p=4$ and 200 sampling points. In the later part of the paper, the procedure given here is employed to obtain the best regression model, and the corresponding convergence results are not given to avoid the repetitiveness of the procedure. While calculating the system responses (eigenvalues) using PCE, a total of 5500 random sampling points are considered. After obtaining the regression model, the probability of occurrence distribution is compared in Fig. 10 with MCS results, which are in good agreement. By comparing Figs. 4 and 10, we can conclude that the dispersion is more when the baffle is present in the liquid tank. The observed coefficient of variation in the first slosh frequency is $5.84 \%$ compared to $5.08 \%$ in Sect. 4.1. This shows that the slosh frequency depends in a complicated way on the uncertainty in the acceleration due to gravity as opposed to the earlier example problem in Sect. 4.1. 


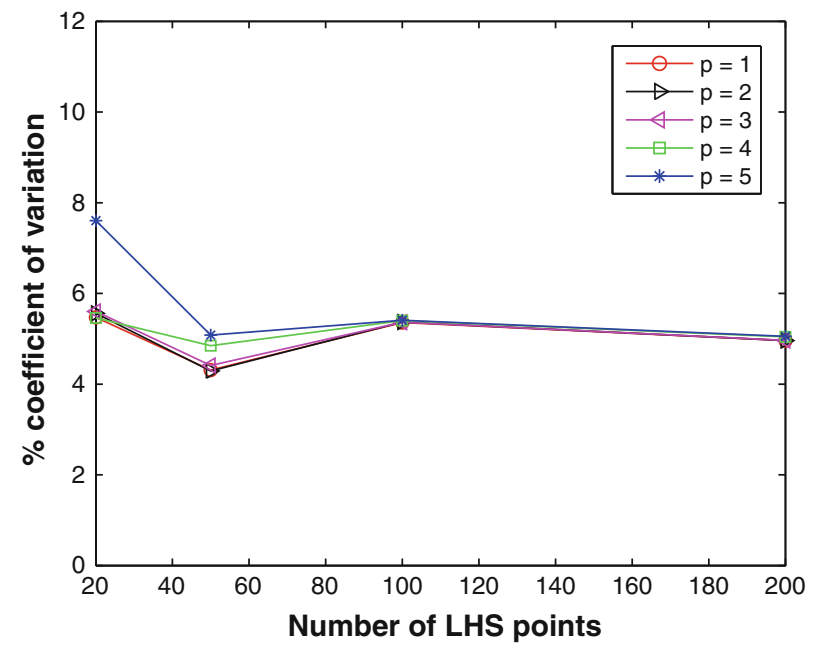

Fig. 9 Convergence of coefficient of variation with the number of LHS points for various polynomial orders

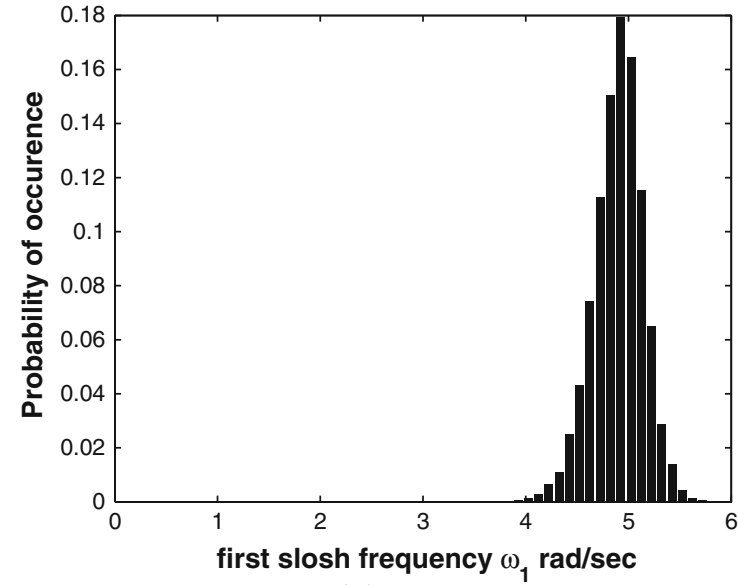

(a) PCE results

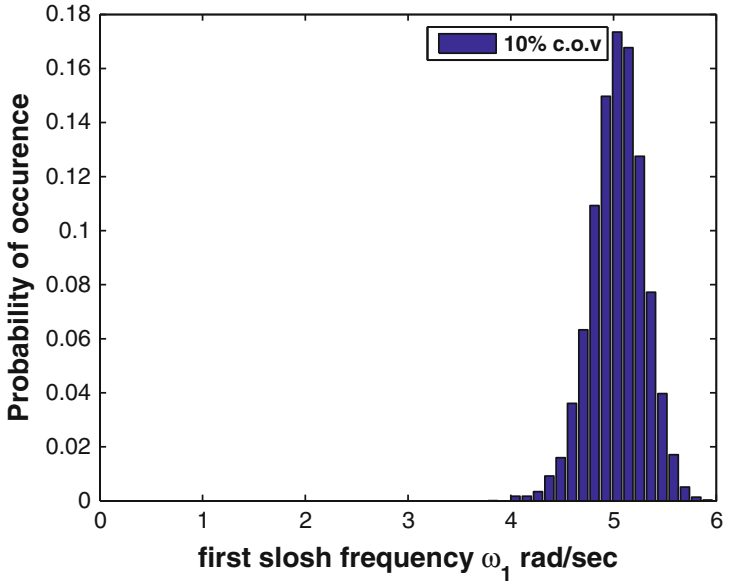

(b) MCS results

Fig. 10 Probability of occurrence distributions of first slosh frequency $\omega_{1}$

\subsubsection{Case 2:}

In the second case, the bulk modulus $(\mathrm{K})$ of the liquid is considered as a random variable with mean value as given in Table 1 and coefficient of variation of 0.05 . The acoustic frequencies are higher-order volume change modes, which cause forces on the bulk of the tank. The effect of the baffle on the acoustic frequencies is complex. Since slosh modes are experienced by the free surface of the liquid, and the penetration of the slosh mode into the liquid column depth depends on the frequency value [3-5], the interaction of the baffle is limited to the volume of the fluid between baffle and the free surface. Whereas, acoustic modes are volume change modes and the effect of the baffle is experienced by the entire fluid volume. These acoustic modes are of importance in tall water columns. In the present case, a total of 20,50,100, 500, and 1,000 LHS points are considered. A convergence test is carried out as done in the previous Sections. The converged regression model is constructed with $p=4$ and 1,000 LHS points. The probability of the occurrence distribution is shown in Fig. 11. While calculating this solution from PCE, 10,000 normally distributed random samples are taken. The same result is compared with a standard MCS result obtained with 10000 random samples, and the agreement is quite good. The coefficient of variation in the first acoustic frequency is $6.4 \%$ which is higher than the considered coefficient of variation (5\%) in the bulk modulus of the fluid. It implies that as mentioned in the beginning of case 2, baffles alter the acoustic frequencies by a considerable amount. 


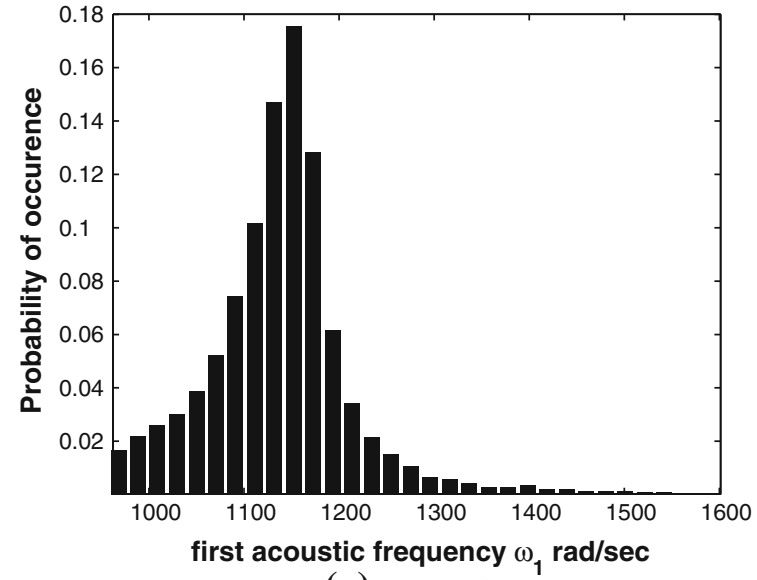

(a) PCE results

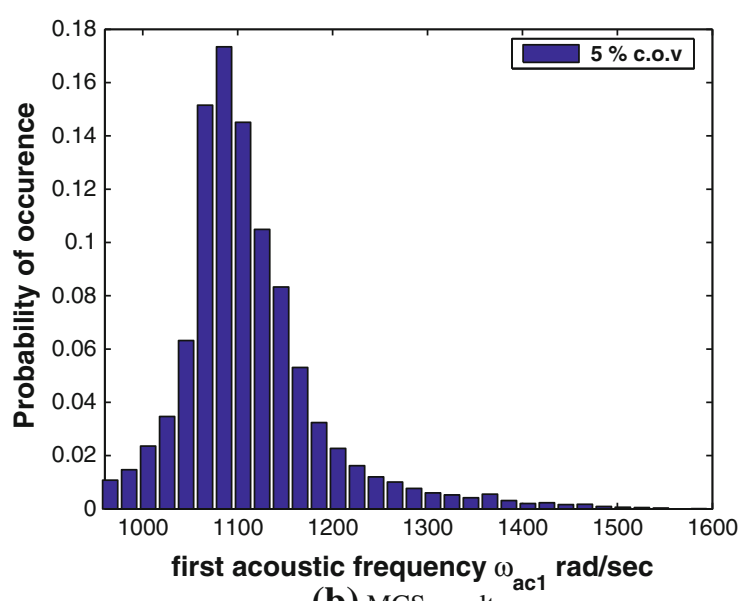

(b) MCS results

Fig. 11 Probability density function of first acoustic frequency $\omega_{1}$

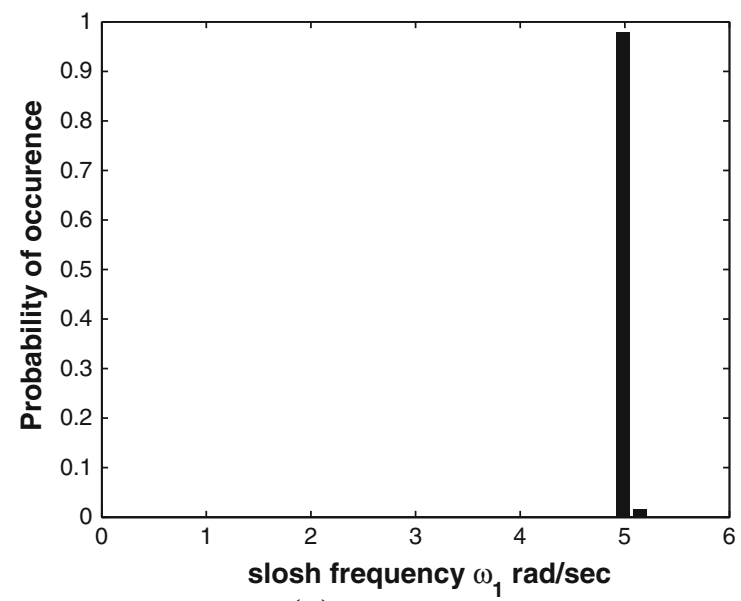

(a) PCE results

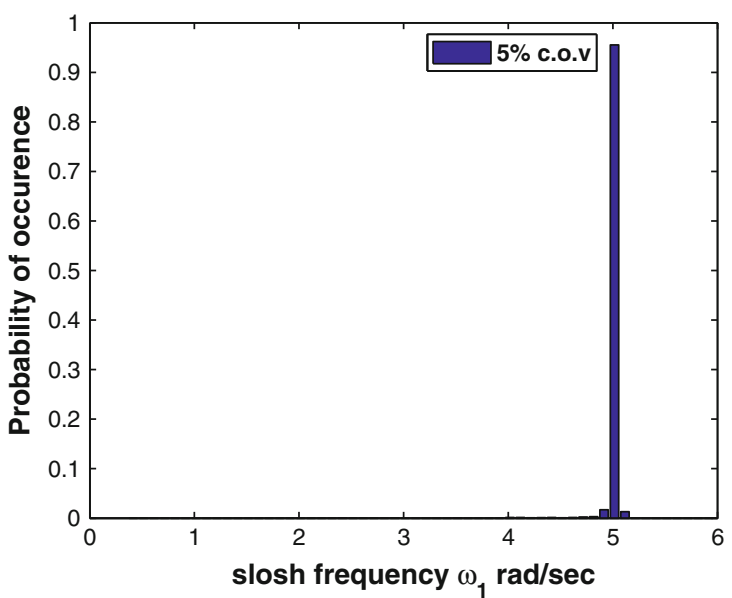

(b) MCS results

Fig. 12 Probability density function of first slosh frequency $\omega_{1}$

\subsubsection{Case 3:}

In the third example case, Young's modulus $(E)$ of the baffle is considered as a random variable with mean $E=62.43 e 9 P a$ [55] and coefficient of variation (c.o.v.) 0.05 . In the present case, a total of 10, 20, 50, and 100 LHS points are considered. The first slosh vibration mode is studied in the present Section with respect to the material (Young's modulus $E$ ) uncertainties in the baffle, since this mode can cause significant lateral forces on the system in which the liquid-filled container is installed. The uncertainties considered in the Young's modulus $(E)$ propagate to the stiffness of the baffle, which in turn changes the damping created by the baffle on the liquid motion.

As explained in the Introduction, slosh frequencies depend on the geometry of the tank, liquid column height, acceleration due to gravity, and density of the liquid. When the baffle is fixed inside a flexible rectangular tank, the dependency of the slosh modes on various parameters will be complex. The converged Hermite polynomial order and number of LHS points are 4 and 100, respectively. The regression model is constructed with $p=4$ and 100 LHS points. The probability of occurrence distribution of the slosh frequency from the present PCE with 10,000 sampling points is given in Fig. 12. The PCE result is also compared with the MCS result in Fig. 12. From Fig. 12, it is clear that the probability of occurrence is high at the center of the distribution and at other frequencies it is very small. The coefficient of variation of the first slosh frequency is $1.68 \%$, and comparing Figs. 10 and 12, it can be observed that the dispersion about the mean in the slosh frequencies is much more due to uncertainties in the acceleration due to gravity ( $\mathrm{g}$ ) compared to uncertainties 


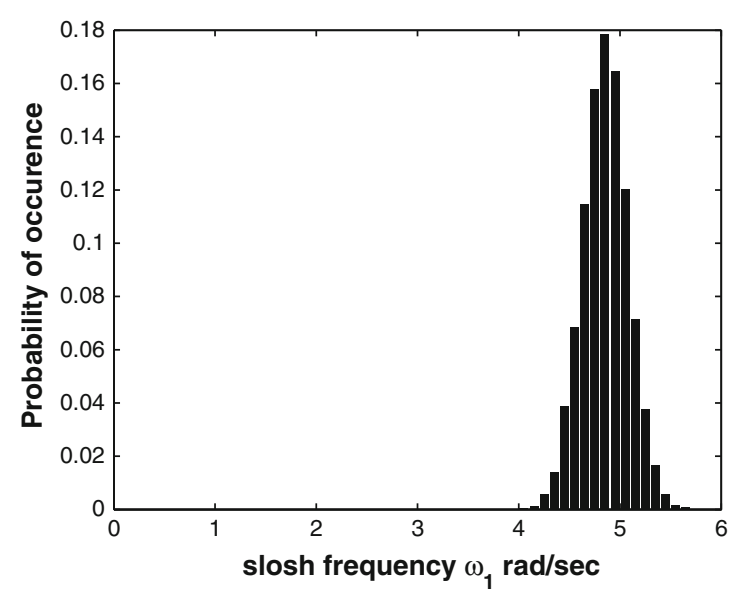

(a) Slosh frequency

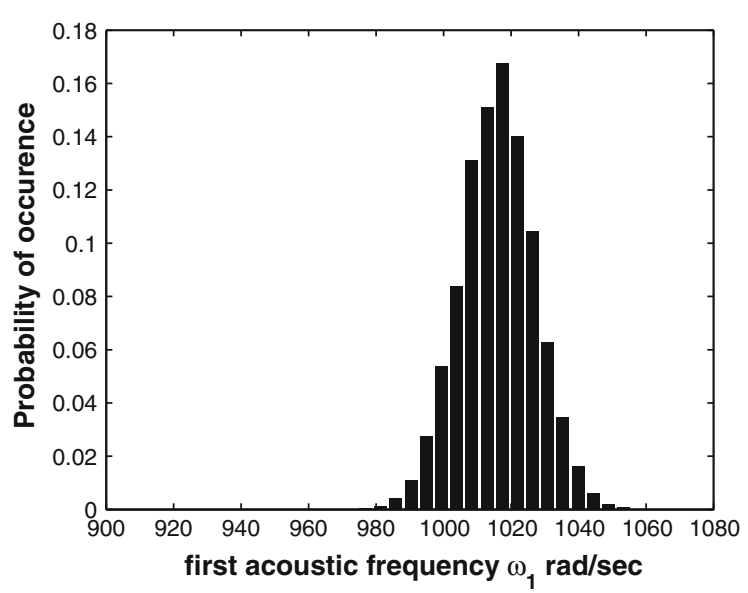

(b) Acoustic frequency

Fig. 13 PCE Probability density functions

in the Young's modulus $(E)$ of the baffle. Also, the coefficient of variation in case 1 is $5.84 \%$ which is higher than in the present case. Hence, it can be concluded that the acceleration due to gravity ( $\mathrm{g}$ ) is a significant random input parameter for the slosh motion in a partially filled liquid rectangular tank with a baffle.

\subsubsection{Case 4:}

In this last example case, acceleration due to gravity (g), bulk modulus of the fluid (K), and Young's modulus $(E)$ of the baffle are considered as uncorrelated random variables simultaneously in the uncertainty analysis. In this case, 3-D polynomial chaos expansion has to be considered corresponding to three random input variables. Assumed coefficients of variations are 0.1 for acceleration due to gravity, and 0.05 for the bulk modulus of the fluid and Young's modulus of the baffle, respectively. For this case also, a rectangular tank with a baffle as considered in Sect 4.2 is taken. This particular case is considered to explore the effect on the slosh and acoustic frequencies when all three random variables are present in the random system. The probability of occurrences of the slosh and acoustic modes are given in Fig. 13. While calculating the probability of occurrences using PCE, 10000 sampling points are considered. Comparing Figs. 10a and 13a, it can be concluded that the uncertainties present in the bulk modulus of the fluid and Young's modulus of the baffle have little effect on the slosh frequencies. The uncertainties present in the three random variables, considered in the present analysis, have a major effect on the acoustic frequencies as clearly seen from Figs. 11a and 13b.

\section{Conclusions}

A computationally efficient method of polynomial chaos expansion with Latin hypercube sampling is employed for uncertainty analysis. The coefficients of the PC expansion models are obtained using LHS points with the least squares method. Convergence on the mean and coefficient of variation of the random system are considered as cut-off criteria for the Hermite polynomial basis function order and number of LHS points. It is concluded from the convergence tests that the Hermite polynomial order $p=4$ is sufficient for the problems considered in the present paper.

The proposed method is applied to uncertainty analysis of sloshing and acoustic frequencies in a rectangular tank with and without a baffle. The significance of uncertainty propagation to the slosh and acoustic frequencies is studied considering acceleration due to gravity, bulk modulus of the fluid, and Young's modulus of the baffle as random processes. It is found that the dispersion in the system response to the considered uncorrelated random variables is more when the baffle is present in the rectangular tank. While the baffle is introduced to dampen the sloshing motion and create desirable mode shift, an increase of dispersion in the eigenvalues may be an undesirable consequence. Moreover, the dispersion in the slosh frequencies is more due to uncertainty in the acceleration due to gravity compared to the uncertainties in the Young's modulus of the baffle. The results obtained from the present PCE procedure are validated against MCS results. The results match well with MCS results, which shows the efficiency and accuracy of the present procedure. 
This efficient and computationally inexpensive method could be a starting point for future research wherein various uncertainty studies can be conducted, which includes uncertainties in the geometry of the baffles, location, material (composite or metal), and density of the baffles.

Acknowledgments The first author would like to acknowledge the John Argyris Foundation for financial support.

\section{References}

1. Sewell, J.L.: An experimental and theoretical study of the effect of fuel on pitching translation flutter. NACA TN 4166 (1957)

2. Graham, E.W., Rodriguez, A.M.: The characteristics of fuel motion which affects airplane dynamics. J. Appl. Mech. 19, 381-388 (1952)

3. Abramson, H.N.: The dynamic behavior of liquids in moving containers. NASA SP-106 (1966)

4. Helmut, F.B.: Theory of the fluid oscillations in a circular cylindrical ring tank partially filled with liquid. NASA TN D-557 (1960)

5. Helmut, F.B.: Fluid oscillations in the containers of a space vehicle and their influence upon stability. NASA TR R-187 (1964)

6. Oberkampf, W.L., Diegert, K.V., Alvin, K.F., Rutherford, B.M.: Variability, uncertainty, and error in computational simulation. AIAA/ASME joint thermophysics and heat transfer conference, ASME-HTD, AIAA, Reston, VA. 357, 259-272 (1998)

7. Du, X., Chen, W.: Methodology for managing the effect of uncertainty in simulation-based design. AIAA J. 38, $1471-1478(2000)$

8. Du, X., Chen, W.: Towards a better understanding of modeling feasibility robustness in engineering design. J. Mech. Des. 122, 357-383 (2000)

9. Zang, T.A., Hemsch, M.J., Hilburger, M.W., Kenny, S.P., Luckring, J.M., Maghami, P., Padula, S.L., Stroud, W.J.: Needs and opportunities for uncertainty-based multidisciplinary design methods for aerospace vehicles, NASA TM-2002-211462, (2002)

10. Hong, Z.H.: Reliability analysis method in the presence of fuzziness attached to operating time. Microelectron. Reliab. 35, 1483-1487 (1995)

11. Jia, G., Xiaoping, D.: Sensitivity analysis with mixture of epistemic and aleatory uncertainties. AIAA J. 45, 2337-2349 (2007)

12. Gayathri, P., Umesh, K., Ganguli, R.: Effect of matrix cracking and material uncertainty on composite plates. Reliab. Eng. Syst. Safety 95, 716-728 (2010)

13. Murugan, S., Harursampath, D., Ganguli, R.: Material uncertainty propagation in helicopter nonlinear aeroelastic response and vibration analysis. AIAA J. 46, 2332-2344 (2008)

14. Murugan, S., Ganguli, R., Harursampath, D.: Aeroelastic analysis of composite Helicopter rotor with random material properties. J. Aircraft 45, 306-322 (2008)

15. Siva, C., Murugan, S., Ganguli, R.: Effect of uncertainty on Helicopter performance prediction. Proc. Inst. Mech. Eng. Part G: J. Aerosp. Eng. 224, 549-562 (2010)

16. Choi, S., Grandhi, R.V., Canfield, R.A.: Reliability-based structural design. Springer, London (2006)

17. Dohnal, F., Mace, B.R., Ferguson, N.S.: Joint uncertainty propagation in linear structural dynamics using stochastic reduced basis methods. AIAA J. 47, 961-969 (2009)

18. Kleiber, M., Hein, T.D.: The stochastic finite element method, basic perturbation technique and computer implementation. pp. 81-145. Wiley, New York (1992)

19. Spanos, P.D., Ghanem, R.G.: Stochastic finite element expansion for random media. J. Eng. Mech. ASCE 115, 1035-1053 (1989)

20. Spanos, P.D., Ghanem, R.G.: Boundary element formulation for random vibration problems. J. Eng. Mech. ASCE 117, 409-423 (1991)

21. Ghanem, R.G., Spanos, P.D.: Polynomial chaos in stochastic finite elements. J. Appl. Mech. ASME 57, 197-202 (1990)

22. Ghanem, R.G., Kruger, R.M.: Numerical solution of spectral stochastic finite element systems. Comput. Methods Appl. Mech. Eng. 129, 289-303 (1996)

23. Ghanem, R.G.: Ingredients for a general purpose stochastic finite element implementation. Comput. Methods Appl. Mech. Eng. 108, 19-34 (1999)

24. William, P.S.: A Monte Carlo dispersion analysis of the X-33 simulation software. AIAA atmospheric flight mechanics conference, August 6-9, Montreal, Canada (2001)

25. Hanson, J.H., Hall, C.E.: Learning about ares I from Monte Carlo simulation. In: AIAA Guidance, Navigation and Control Conference and Exhibit, 18-21, Honolulu, Hawaii (2008)

26. Chen, L.C., Zhu, W.Q.: Stochastic stability of Duffing oscillator with fractional derivative damping under combined harmonic and white noise parametric excitations. Acta Mech. 207, 109-120 (2009)

27. Chen, L.C., Deng, M.L., Zhu, W.Q.: First passage failure of quasi integrable-Hamiltonian systems under combined harmonic and white noise excitations. Acta Mech. 206, 133-148 (2009)

28. Schueller, G.I., Pradlwarter, H.J.: Uncertainty analysis of complex structural systems. Int. J. Numer. Methods Eng. 80, 881-913 (2009)

29. Rao, S.S., Berke, L.: Analysis of uncertain structural systems using interval analysis. AIAA J. 35, $727-735$ (1997)

30. Zadeh, L.: Fuzzy sets. Inform. Control 8, 338-353 (1965) 
31. Rao, S.S., Sawyer, J.P.: Fuzzy finite element approach for the analysis of imprecisely defined systems. AIAA J. 33, 2364-2370 (1995)

32. David, M., Dirk, V.: Fuzzy finite element method for frequency response function analysis of uncertain structures. AIAA J. 40, 126-136 (2002)

33. Qing, L., Rao, S.S.: Fuzzy finite element approach for analysis of fiber-reinforced laminated composite beams. AIAA J. 43, 651-661 (2005)

34. Klir, G.J., Wierman, M.J.: Uncertainty-based information elements of generalized information theory. Physica, Heidelberg (1999)

35. Klir, G.J.: Generalized information theory: aims, results, and open problems. Reliab. Eng. Syst. Safety 85, 21-38 (2004)

36. Mourelatos, Z., Zhou, J.: Reliability estimation and design with insufficient data based on possibility theory. AIAA J. 43, 1696-1705 (2005)

37. Shafer, G.: A mathematical theory of evidence. Princeton University Press, Princeton, NJ (1976)

38. Oberkampf, W.L., Helton, J.C.: Investigation of evidence theory for engineering applications. In: 43rd AIAA/ASME/ ASCE/AHS/ASC Structures, Structural Dynamics, and Materials Conference, 4th Non-Deterministic Approaches Forum, AIAA Paper 2002-1569 (2002)

39. Bae, H.R., Ramana, V.G., Robert, A.C.: Uncertainty quantification of structural response using evidence theory. AIAA J. 41, 2062-2068 (2003)

40. Rao, S.S., Annamdas, K.: Evidence-based fuzzy approach for the safety analysis of uncertain systems. AIAA J. 46, 2383-2387 (2008)

41. Seung, K.C., Ramana, V.G., Robert, A.C., Chris, L.P.: Polynomial chaos expansion with latin hypercube samplings for estimation response variability. AIAA J. 42, 191-1198 (2004)

42. Wei, D.L., Cui, Z.S., Chen, J.: Uncertainty quantification using polynomial chaos expansion with points of monomial cubature rules. Comput. Struct. 86, 2102-2108 (2008)

43. Dongbin, X., Karniadakis, G.M.: Modeling uncertainty in steady state diffusion problems via generalized polynomial chaos. Comput. Methods Appl. Mech. Eng. 191, 4927-4948 (2002)

44. Simon, F., Guillen, P., Sagaut, P., Lucor, D.: A gPC-based approach to uncertain transonic aerodynamics. Comput. Methods Appl. Mech. Eng. 199, 1091-1099 (2010)

45. Iannotta, B.: Slosh, rattle and roll. New Scientist; May 27, 32-35 (2000)

46. Gerrits, J., Veldman, A.E.P.: Dynamics of liquid-filled spacecraft. J. Eng. Math. 45, 21-38 (2003)

47. Traugott, S.C., Mehta, G.: Response of small amplitude slosh to time-dependent effective gravity. AIAA-1984-1424 SAE, and ASME, Joint Propulsion Conference, 20th, Cincinnati, OH, June 11-13, (1984)

48. Hung, R.J., Long, Y.T., Chi, Y.M.: Slosh dynamics coupled with spacecraft attitude dynamics. I - Formulation and theory. J. Spacecraft Rockets 33, 575-581 (1996)

49. Alireza, B., Hari, B.V.: Prediction of bulk modulus and volumetric expansion coefficient of water for leak tightness test of pipelines. Int. J. Pressure Vessels Piping 86, 550-554 (2009)

50. Krishna, K.D., Gopalakrishnan, S., Ganguli, R.: Three dimensional sloshing: A consistent finite element formulation. Int. J. Numer. Methods Fluids 2010. doi:10.1002/fld.2260

51. Wiener, N.: The homogeneous chaos. Am. J. Math. 60, 897-939 (1938)

52. Thierry, C., Olivier, L.M., Martinez, J.M.: Polynomial chaos expansion for sensitivity analysis. Reliab. Eng. Syst. Safety 94, 1161-1172 (2009)

53. Cole, H.A., Gambucci, B.J.: Measured two-dimensional damping effectiveness of fuel sloshing baffles applied to ring baffles in cylindrical tanks, NASA TN D-694 (1961)

54. Silveira, M.A., Stephens, D.G., Leonard, H.W.: Damping of liquid oscillations in cylindrical tanks with various baffles, NASA TN D-1709 (1963)

55. Alfredo, B., Rodolfo, R., Durate, S.: Finite element computation of sloshing modes in containers with elastic baffle plates. Int. J. Numer. Methods Eng. 56, 447-467 (2003)

56. Satish, C., Prathap, G.: A field consistent formulation for the eight noded solid finite element. Comput. Struct. 33, 345-355 (1989) 